\title{
Stability-integrated Fuzzy $C$ means segmentation for spatial incorporated automation of number of clusters
}

\author{
V ROYNA DAISY ${ }^{1, *}$ and S NIRMALA ${ }^{2}$ \\ ${ }^{1}$ Department of Electrical and Electronics Engineering, Paavai Engineering College, Namakkal 637 018, India \\ ${ }^{2}$ Department of Electronics and Communication Engineering, Muthayammal Engineering College, \\ Rasipuram 637 408, India \\ e-mail: roynadaisy@gmail.com; roynavedhanayagampec@paavai.edu.in; nirmala.ramkamal@gmail.com
}

MS received 2 December 2016; revised 18 February 2017; accepted 21 August 2017; published online 16 March 2018

\begin{abstract}
Fuzzy $C$ Means clustering, one of the predominant segmentation algorithms, requires prior knowledge of number of clusters in the image and is sensitive to noise and outliers. Determining the number of clusters and including spatial information to basic Fuzzy $C$ Means clustering are done in numerous ways. Literature reveals that either number of clusters is defined or spatial information is incorporated. In the proposed work, spatial information and cluster determination are integrated based on the concept of stability. Implementation of split and merge algorithm to find the number of clusters is done based on the modified Sylvester's theorem in the context of positive definite functions. Experiments are performed on synthetic and real images and the number of clusters determined is validated using validation indices. Results show that correct clusters are classified with robustness to noise.
\end{abstract}

Keywords. Fuzzy $C$ Means clustering; stability; positive definite functions; spatial information; validity index.

\section{Introduction}

Magnetic Resonance Imaging (MRI) is a vital diagnostic procedure used for analysing the neurological disorders that cause changes in the shape, thickness, mass and volume of brain tissues. Image processing is done in order to make quantitative and qualitative measurement of the alteration made to analyse and classify the disorders. The most crucial step in the process of image processing is image segmentation. Different image segmentation algorithms have been developed, yet each method has its own advantages and limitations.

The Fuzzy $C$ Means (FCM) clustering, one of the powerful clustering techniques shown in literature, is a popular unsupervised classification method and has found many applications in pattern classification and image segmentation [1-5]. The application of clustering algorithms is constrained with its dependence on parameter setting and initialization. Although FCM is prominent for image segmentation, the results are mainly affected by the identification of number of clusters and the initialization of the centroids. In case of brain MRI, normally we go for segmenting the white matter (WM), grey matter (GM) and cerebro-spinal fluid (CSF) and hence the number of clusters can be set to three. However, in case of neurological disorders there are possibilities for tumours, lesions or blurring

*For correspondence of normal tissues where we cannot predetermine the number of clusters. If the number of clusters is not set to the number of natural clusters or the centroids are initialized randomly, the clustering results would be unreliable and inconsistent. Hence, for automatic segmentation using FCM, clustering several techniques has been developed by determining the number of clusters [6-9].

A few algorithms that determine the cluster number have been proposed in the literature. A variety of self-splittingmerging clustering algorithms have been developed for determining the number of clusters. Zhang and Liu [10] proposed a self-splitting algorithm for clustering where the number of initial clusters is set to a minimum of two and the clusters are split based on a score function until the number of clusters reaches a predefined maximum. Sun et al [11] proposed a model-based selection algorithm, which involves high computational complexity as the splitting involves calculation of distance of all data vectors in the cluster with the remaining cluster centre. Lin and Chen [12] proposed a combinatorial splitting algorithm with cohesion merging. Splitting one cluster at a time will lead to high computational time. Hence, a multiple splitting algorithm was proposed by Liu and Ramamohanarao [13]. Yang et al [14] and RuiFa et al [15] have proposed automatic detection of cluster numbers. However, these methods involve distance calculation, which increases the computational time of FCM algorithm. Zanaty [16] 
proposed a method of reducing the computational overhead due to distance calculation by replacing the Euclidean distance with a Gaussian radial basis classifier.

The basic FCM is highly susceptible to noise as the algorithm does not consider the spatial information during clustering. This is again a highly projected area where several algorithms have been proposed involving spatial information in order to make the segmentation insensible to noise. Chuang et al [17] proposed a method that incorporates spatial information, where based on the cluster distribution in the neighbourhood the weighting function is altered thus resulting in segmentation with homogeneous regions. When the images are highly contaminated by noise the neighbouring pixels of a pixel may also contain abnormal features. Hence segmentation done with spatial information in the local region may not yield good results. Zhao et al [18] proposed non-local spatial information segmentation as many pixels possess a similar neighbourhood configuration over the entire image domain the local spatial constraint is being replaced by non-local spatial constraint. Zaixin et al [19] introduced the impact of image patches and local statistics, proposing a novel similarity measure, and variation was done in the objective function by replacing the Euclidean distance by neighbourhood-weighted distance. Zhang et al [20] utilize Gaussian distribution for distance calculation and for estimating the prior probability, the hidden Markov random field is replaced by mean template. Yanling and Yi [21] incorporate pixel probability and neighbourhood statistics into the membership function. Gong et al [9] modify the objective function with Kernel distance function and make the algorithm parameter independent.

There are several techniques proposed for determining the cluster number for segmentation of distinct regions and work has been done for making the segmentation insensitive to noise by incorporating spatial constraints of different types. But still there is no method of integrating detection of the number of clusters with spatial information of pixels within the image. An integrated technique based on the concept of stability is proposed to make the FCM segmentation process robust. The proposed algorithm is used to find the number of clusters in conjunction with incorporating the spatial information. Sylvester's theorem for positive definite functions [22], [23] is slightly modified and applied to find the region that is not convergent with the region of convergence (ROC). This method of segmentation is region based and hence does not allow oversegmentation or erroneous results due to noisy pixels. Also the problem of seed selection is rectified as the cluster centres are taken as the seed pixel.

The organization of paper includes the modified Sylvester's theorem for positive definite function in section 2 , and section 3 presents the validity indices for determining the number of clusters. The modification of FCM algorithm with the concept of positive, semi-positive and negative definite functions is explained and its applications to image segmentation is explained in section 4 . Section 5 includes discussion on the experimental results compared to other methods and section 6 gives the conclusion.

\section{Modified Sylvester's method}

Sylvester's method is used for testing whether the given function is positive definite or not. Positive definite concept is used for checking whether a system is stable or not. Consider a system that is expressed as a matrix

$$
\left[\begin{array}{lll}
a_{11} & a_{12} & a_{13} \\
a_{21} & a_{22} & a_{23} \\
a_{31} & a_{32} & a_{33}
\end{array}\right]
$$

By Sylvester's theorem, a function is positive definite when

$$
\begin{aligned}
& \left|\begin{array}{ll}
\left|a_{11}\right|>0 \\
a_{11} & a_{12} \\
a_{21} & a_{22}
\end{array}\right|>0 \Rightarrow a_{11} a_{22}-a_{12} a_{21}>0 \\
& \left|\begin{array}{lll}
a_{11} & a_{12} & a_{13} \\
a_{21} & a_{22} & a_{23} \\
a_{31} & a_{32} & a_{33}
\end{array}\right|>0 \Rightarrow a_{11}\left(a_{22} a_{33}-a_{23} a_{32}\right) \\
& -a_{12}\left(a_{21} a_{33}-a_{31} a_{23}\right)+a_{13}\left(a_{21} a_{32}-a_{22} a_{31}\right)>0 \\
& \mid \begin{array}{l}
a_{11} \mid>0 \\
\left|\begin{array}{ll}
a_{11} & a_{12} \\
a_{21} & a_{22}
\end{array}\right|>0 \\
\left|\begin{array}{lll}
a_{11} & a_{12} & a_{13} \\
a_{21} & a_{22} & a_{23} \\
a_{31} & a_{32} & a_{33}
\end{array}\right|>0 \Rightarrow a_{11} a_{22}-a_{12} a_{21}>0 \\
-a_{12}\left(a_{21} a_{33}-a_{31} a_{23}\right)+a_{13}\left(a_{21} a_{32}-a_{22} a_{31}\right)>0
\end{array}
\end{aligned}
$$

If all the three conditions are satisfied, then the given function is positive definite. If any of the three conditions is not satisfied, then the given function is negative definite. This concept of Sylvester's theorem is applied in image segmentation to find asymptotic stability. However, the problem with the application of original Sylvester's theorem is that these three conditions for positive definiteness of a homogeneous grey, black and white regions will result in erroneous conclusion. The result obtained for the second and third conditions will be the same for all homogeneous, grey, black and white regions. Hence for the application to segmentation problem, the Sylvester's theorem has been modified slightly to incorporate the changes in the different homogeneous images.

$$
\begin{aligned}
& \left|\begin{array}{ll}
a_{11} \mid>0 \\
a_{11} & a_{12} \\
a_{21} & a_{22}
\end{array}\right|>0 \Rightarrow a_{11} a_{22}+a_{12} a_{21}>0 \\
& \left|\begin{array}{lll}
a_{11} & a_{12} & a_{13} \\
a_{21} & a_{22} & a_{23} \\
a_{31} & a_{32} & a_{33}
\end{array}\right|>0 \Rightarrow a_{11}\left(a_{22} a_{33}+a_{23} a_{32}\right) \\
& -a_{12}\left(a_{21} a_{33}+a_{31} a_{23}\right)+a_{13}\left(a_{21} a_{32}+a_{22} a_{31}\right)>0
\end{aligned}
$$


This slight modification in Sylvester's theorem will eliminate the problem of misinterpretation when applied in segmentation.

\section{Validity indexes for determination of cluster number}

Image segmentation by FCM clustering shows good segmentation accuracy. However, fuzzy clustering has an important issue of requiring prior knowledge of the data or the number of clusters in the data. Prediction of number of clusters for new dataset is highly sensitive in terms of segmentation accuracy. There are several validity indexes proposed in the literature under several categories. Different types of indexes are defined based on only membership values, based on membership matrix and original dataset and finally based on statistical model selection. Among the several validity indexes, a few indexes are defined upon which the cluster accuracy is analysed.

\subsection{Partition coefficient}

Bezdek et al [24] proposed Partition Coefficient (PC) expressed as

$$
P C=\frac{1}{N} \sum_{i=1}^{N} \sum_{j=1}^{C} u_{i j}^{2} .
$$

The PC index ranges from $1 / C$ to unity. No clustering probability prevails if the index nears $1 / C$. Hard clusters are obtained when index value approximates near unity.

\subsection{Partition entropy coefficient}

Bezdek et al [24] defined another validity index, Partition Entropy Coefficient (PE), based on the membership values:

$$
P E=\frac{1}{N} \sum_{i=1}^{N} \sum_{j=1}^{C} u_{i j} \cdot \log \left(u_{i j}\right)
$$

The index value ranges in $[0, \log C]$. Hard clusters are obtained as the PE value nears the lower limit (0). The upper limit $(\log C)$ of index exhibits the lack of ability to cluster.

\subsection{Xie-Beni index}

Indexes based on membership values (PC and PE) exhibit monotonous dependence on the number of clusters, sensitivity to fuzzifying factor $m$ and lack of direct connection to the geometry of data. Xie-Beni index (XB) [25] is a category of validity index based on membership values and dataset. The $\mathrm{XB}$ index, also called as the compactness and separation validity function, is a well-performing validity index for fuzzy clustering. $\mathrm{XB}$ index is defined as

$$
X B=\frac{\left\{\left(\frac{1}{N}\right) \sum_{i=1}^{C} \sigma_{i}^{2}\right\}}{\left\{D_{\min }\right\}^{2}}
$$

where

$$
\sigma_{i}^{2}=\sum_{j=1}^{N} u_{i j}\left\|x_{j}-c_{i}\right\|^{2} .
$$

The number of feature vectors is denoted as $x_{j}$ for $j=1,2, \ldots, N$. The vector $x_{j}$ is to be clustered into $c_{i}$ clusters where $i=1,2, \ldots, C$. $D_{\min }$ is the minimum distance between the cluster centres. The fuzzy weighted mean square error $\sigma_{i}^{2}$ varies based on the compactness of clusters. For hard clusters, $\sigma_{i}^{2}$ is smaller. Small values of XB are obtained for compact and well-separated clusters.

\subsection{Fukuyama-Sugeno index}

The Fukuyama-Sugeno index (FS) [26] is defined as

$$
F S=\sum_{j=1}^{N} \sum_{i=1}^{C} u_{i j}^{m}\left(\left\|x_{j}-c_{i}\right\|^{2}-\left\|c_{i}-\bar{c}\right\|^{2}\right)
$$

where

$$
\bar{c}=\sum_{i=1}^{C} \frac{c_{i}}{C} .
$$

The FS calculation is based on the compactness of the clusters and the distance between the two clusters. For wellseparated and compact clusters, the FS values are small.

\subsection{Average partition density}

The average partition density (PA) [27] is defined as

$$
P A=\frac{1}{C} \sum_{i=1}^{C} \frac{S_{i}}{V_{i}}
$$

where $S_{i}=\sum_{x \in x_{i}} u_{i j}$ is sum of central members of cluster $i$ and $C$ is the number of clusters. For compact clusters the PA value should be large.

\subsection{Partition density index}

The partition density index (PD) [27] is defined as

$$
P D=\frac{\sum_{i=1}^{C} S_{i}}{F H}
$$


Table 1. Number of clusters by the validity indexes.

\begin{tabular}{lrrrrrrrr}
\hline $\begin{array}{l}\text { Type of } \\
\text { image }\end{array}$ & $\begin{array}{c}\text { Noise level } \\
(\%)\end{array}$ & PC & PE & XB & FS & PA & PD & AIC \\
\hline Synthetic & 0 & 4 & 3 & 3 & 3 & 4 & 3 & 3 \\
$\quad$ image 1 & 6 & 3 & 3 & 5 & 3 & 4 & 6 & 3 \\
& 12 & 5 & 6 & 6 & 3 & 3 & 5 & 3 \\
Synthetic & 0 & 4 & 4 & 4 & 5 & 4 & 5 & 4 \\
image 2 & 6 & 4 & 5 & 5 & 4 & 6 & 4 & 4 \\
& 12 & 5 & 5 & 4 & 4 & 6 & 4 & 4 \\
Brain data 1 & 0 & 10 & 10 & 10 & 13 & 10 & 11 & 10 \\
& 5 & 12 & 10 & 10 & 10 & 12 & 10 & 10 \\
Brain data 2 & 10 & 14 & 15 & 14 & 10 & 10 & 11 & 10 \\
& 0 & 14 & 12 & 14 & 12 & 12 & 14 & 12 \\
& 5 & 12 & 12 & 16 & 12 & 16 & 14 & 12 \\
& 10 & 16 & 14 & 12 & 16 & 15 & 12 & 12 \\
\hline
\end{tabular}

where the Fuzzy Hyper-Volume (FH) is proposed by Gath and Geva [28] based on the concepts of hyper-volume and density:

$$
F H=\sum_{i=1}^{C} V_{i}
$$

and

$$
V_{i}=\left(\frac{\sum_{j=1}^{N} u_{i j}^{m}\left(x_{j}-c_{i}\right)\left(x_{j}-c_{i}\right)^{T}}{\sum_{j=1}^{N} u_{i j}^{m}}\right)^{1 / 2}
$$

The PD value should be high for compact clusters.

\subsection{Akaike's information criterion index}

The index based on Akaike's information criterion (AIC) [29] is defined as

$$
A I C=D_{a}-2 \mu \sigma^{2}
$$

where $\mu(C)$ is the number of degree of freedom of the model:

$$
\text { for soft clusters } \mu(C)=(C-1) N+C
$$

$$
\text { for hard clusters } \mu(C)=N+C \text {. }
$$

The average separation between two clusters over all possible pairs of clusters is

$$
D_{a}=\frac{1}{N} \sum_{i=1}^{C} \sum_{x \in v_{i}}\left\|x-c_{i}\right\|^{2}
$$

and noise level $\sigma$ is obtained from the relation

$$
\sigma^{2}=\frac{D_{a}\left(C^{*}\right)}{\rho N-\mu\left(C^{*}\right)}
$$

where $C^{*}$ is the maximum number of clusters and $\rho$ is the co-dimension of the model $(\rho=1)$.

The AIC index is a statistical model-based validity index. For better clustering results the value of AIC should be small.

The validity indexes are used to check the correct number of clusters. The segmentation results of different algorithms are measured based on a comparison score [30] defined as

$$
S=\frac{\left|A \cap A_{r e f}\right|}{\left|A \cup A_{r e f}\right|}
$$

where $A$ is the set of all pixels belonging to a class based on the algorithm performed and $A_{\text {ref }}$ is the reference cluster pixels.

\section{Proposed method}

The FCM clustering has a vital role in image segmentation. In spite of having several advantages in its own form, still it has the limitation of requiring the number of clusters in advance. Also the basic FCM does have the involvement of spatial information, thus making it sensitive to noise and outliers. Thus the proposed work involves changes in the fundamental FCM algorithm by integrating the stability concepts.

\subsection{Basic FCM algorithm}

FCM has its own meritorious place in the field of image segmentation and pattern recognition. The FCM algorithm partitions every image pixel into a collection of the fuzzy clusters by minimizing the weighted sum of squared error objective function. The objective function of FCM is

$$
J_{m}=\sum_{i=1}^{C} \sum_{j=1}^{N} u_{i j}^{m}\left\|x_{j}-c_{i}\right\|^{2}
$$

Fuzzy partitioning is done iteratively by updating the membership function $u_{i j}$ and cluster centres $C_{i}$ optimizing the objective function. The membership function is updated by

$$
u_{i j}=\frac{1}{\sum_{k=1}^{C}\left(\frac{\left\|x_{j}-c_{i}\right\|}{\left\|x_{j}-c_{k}\right\|}\right)^{2 /(m-1)}}
$$

The cluster centres are updated using 
Table 2. Validity indexes for different images.

\begin{tabular}{|c|c|c|c|c|c|c|c|c|}
\hline Type of image & Noise level (\%) & $\mathrm{PC}$ & $\mathrm{PE}$ & $\begin{array}{c}\mathrm{XB} \\
\left(\times 10^{-3}\right)\end{array}$ & FS & PA & PD & AIC \\
\hline \multirow[t]{3}{*}{ Synthetic image 1} & 0 & 0.7852 & 0.1512 & 2.11 & -21.3526 & 2.9892 & 3.5426 & 0.5876 \\
\hline & 6 & 0.9526 & 0.1938 & 3.65 & -19.6598 & 1.2321 & 0.6858 & 0.5378 \\
\hline & 12 & 0.6258 & 0.5456 & 4.26 & -04.3647 & 3.5467 & 1.2374 & 0.6256 \\
\hline \multirow[t]{3}{*}{ Synthetic image 2} & 0 & 0.9265 & 0.1295 & 1.92 & -12.5874 & 3.6023 & 2.2359 & 0.5278 \\
\hline & 6 & 0.9187 & 0.3515 & 2.84 & -25.6489 & 2.9782 & 3.4937 & 0.5527 \\
\hline & 12 & 0.6528 & 0.4227 & 2.12 & -27.6548 & 2.6845 & 3.6026 & 0.5662 \\
\hline \multirow[t]{3}{*}{ Brain data 1} & 0 & 0.9221 & 0.1824 & 1.89 & -10.2546 & 3.6302 & 2.5291 & 1.4728 \\
\hline & 5 & 0.7542 & 0.1958 & 2.24 & -18.2659 & 1.9827 & 3.2659 & 1.2978 \\
\hline & 10 & 0.6528 & 0.2936 & 3.67 & -20.3268 & 3.5978 & 2.8613 & 1.7892 \\
\hline \multirow[t]{3}{*}{ Brain data 2} & 0 & 0.7296 & 0.1925 & 3.58 & -25.3214 & 3.6152 & 1.9246 & 1.6289 \\
\hline & 5 & 0.9364 & 0.2051 & 4.26 & -23.9584 & 1.5462 & 1.6729 & 1.6918 \\
\hline & 10 & 0.6189 & 0.4126 & 2.15 & -08.3654 & 2.2897 & 3.2984 & 1.7725 \\
\hline
\end{tabular}

$$
c_{i}=\frac{\sum_{j=1}^{N} u_{i j}^{m} x_{j}}{\sum_{j=1}^{N} u_{i j}^{m}} .
$$

The basic FCM algorithm is as follows:

(1) Input the number of clusters $c$, the fuzzifier $m$ and the distance function.

(2) Initialize the cluster centres $c_{i}^{0}(i=1,2,:::, c)$.

(3) Calculate $u_{i j}(j=1,2,:::, n ; i=1,2,:::, c)$ using Eq. (19).

(4) Update $c_{i}(i=1,2,:::, c)$ using Eq. (20).

(5) If $\max _{i . j}\left\|u_{i j}^{t}-u_{i j}^{t-1}\right\| \leq \varepsilon$, then go to step 6 , else go to step 3.

(6) Output the results.

\subsection{Stability criteria for FCM}

The FCM algorithm is made automatic in determining the number of clusters. To segment a region in the image a seed pixel in the region is chosen. A region of convergence (ROC) is formed around the seed pixel by forming a matrix.

The ROC is

$$
\left[\begin{array}{ccc}
c_{11} & c_{12} & c_{13} \\
c_{21} & c_{0} & c_{23} \\
c_{31} & c_{32} & c_{33}
\end{array}\right]
$$

where $c_{0}$ is the cluster centre or seed pixel and $c_{11}, \ldots \ldots, c_{33}$ are pixels adjacent to the cluster centre.

This ROC is taken as the seed region to be segmented. The segmentation process is started by forming regions of adjacency with ROC. The regions of adjacency are formed by forming matrices in the horizontal and vertical directions

The regions of adjacency are formed and the modified Sylvester's theorem for positive definiteness is applied to test whether the centre pixel of each adjacency matrix is asymptotically stable or marginally stable or unstable.

Consider the region of adjacency (ROA) $\left[\begin{array}{lll}x_{11} & x_{12} & x_{13} \\ x_{21} & x_{22} & x_{23} \\ x_{11} & x_{12} & x_{13}\end{array}\right]$ to test for the stability of the matrix centre element $x_{22}$. By applying modified Sylvester's theorem, a check for positive definiteness is performed.

$$
\begin{gathered}
X \equiv c_{11}=x_{11} \pm 0.1 \\
Y \equiv c_{11} c_{0}+c_{12} c_{21}=\left(x_{11} x_{22}+x_{12} x_{21}\right) \pm 0.1 \\
Z \equiv c_{11}\left(c_{0} c_{13}+c_{23} c_{12}\right)+c_{12}\left(c_{21} c_{13}+c_{11} c_{23}\right) \\
+c_{13}\left(c_{21} c_{12}+c_{0} c_{11}\right) \\
=x_{11}\left(x_{22} x_{13}+x_{23} x_{12}\right)+x_{12}\left(x_{21} x_{13}+x_{11} x_{23}\right) \\
+x_{13}\left(x_{21} x_{12}+x_{22} x_{11}\right) \pm 0.1
\end{gathered}
$$

A scaling about $10 \%$ of ROC from ROA is arbitrarily chosen as the MRI image is heterogeneous. Based on these conditions $X, Y, Z$ a procedure for determining the stability of the pixel with the ROC is derived.

(1) XYZ-If all the three conditions are satisfied, then the matrix considered is positive definite and hence the centre element is asymptotically stable, which indicates that it belongs to the ROC.

(2) $\bar{X} Y Z$ - If the first condition is not satisfied but the second and third conditions are satisfied then the matrix considered is positive semi-definite and hence the centre element is marginally stable, which indicates that it forms the edge layer between two distinguished regions

(3) $\bar{X} \bar{Y} \bar{Z}$ - If all three conditions are not satisfied then the matrix considered is negative definite and hence the centre element is unstable, which indicates that it does not belong to the ROC.

(4) $X[Y \bar{Z}+\bar{Y} Z]$ - If the first condition is satisfied but the second or third condition is not satisfied then it 
Table 3. Comparison scores in $\%$ of different algorithms.

\begin{tabular}{lccccc}
\hline $\begin{array}{l}\text { Type of } \\
\text { image }\end{array}$ & $\begin{array}{c}\text { Noise } \\
\text { level }(\%)\end{array}$ & FCM & KFCM & FBSA & $\begin{array}{c}\text { Proposed } \\
\text { method }\end{array}$ \\
\hline Synthetic & 0 & 99.04 & 97.89 & 98.23 & 98.54 \\
image 1 & 6 & 91.56 & 93.45 & 95.20 & 97.34 \\
& 12 & 88.56 & 90.25 & 89.34 & 94.56 \\
Synthetic & 0 & 98.24 & 97.56 & 97.63 & 98.09 \\
$\quad$ image 2 & 6 & 90.27 & 92.34 & 92.05 & 93.89 \\
& 12 & 87.38 & 90.45 & 90.02 & 92.83 \\
Brain data 1 & 0 & 95.23 & 92.56 & 91.93 & 93.10 \\
& 5 & 85.56 & 89.54 & 88.56 & 90.34 \\
Brain data 2 & 10 & 82.43 & 88.35 & 89.29 & 91.28 \\
& 0 & 95.02 & 93.67 & 92.34 & 92.98 \\
& 5 & 87.23 & 91.34 & 90.67 & 92.01 \\
& 10 & 85.34 & 89.32 & 89.26 & 90.38 \\
\hline
\end{tabular}

indicates that the centre element of the matrix considered is a noisy pixel.

\subsection{Stability-based membership function}

It is important to note that the neighbouring pixels in an image are highly correlated and have a high probability of belonging to the same cluster. It becomes necessary to consider the neighbouring pixels while clustering. Also segmentation accuracy of an algorithm mainly depends on the proper segmentation of its edges. This necessitates a concept for analysing the edges while segmenting an image. The spatial function $h_{i j}$ proposed for noisy image segmentation by Chuang et al [17] has been utilized in the proposed work. The spatial function is defined as the summation of the membership functions in the neighbourhood of each pixel under consideration:

$$
h_{i j}=\sum u_{k i}, k \in \mathrm{NB}\left(x_{j}\right)
$$

where $\mathrm{NB}\left(x_{j}\right)$ represents a square window of size $3 \times 3$ centred on pixel $x_{j}$ in the spatial domain. The value of spatial function is large if the majority of its neighbourhood belongs to the same cluster. The spatial function decides whether the pixel belongs to the cluster or not. It does not consider about the edges while forming the clusters. The stability concept explained in section 4.2 has been incorporated to determine the spatial function. The membership value $u_{k i}$ based on stability concept is defined as follows:

$$
\begin{array}{ll}
u_{k i}=1, & S \Rightarrow X Y Z \\
0.5, & S \Rightarrow \bar{X} Y Z \\
0.75, & S \Rightarrow X[Y \bar{Z}+\bar{Y} Z] \\
0, & S \Rightarrow \bar{X} \bar{Y} \bar{Z} .
\end{array}
$$

When $S \Rightarrow X Y Z$, the pixel under consideration has the highest probability of being the member of the cluster and hence has a maximum membership function. Similarly when $S \Rightarrow \bar{X} \bar{Y} \bar{Z}$, the pixel under consideration has the least probability of being the member of the cluster and hence has a minimum membership function. The probability that the pixel under consideration may be a noisy pixel is obtained when $S \Rightarrow X[Y \bar{Z}+\bar{Y} Z]$ is obtained. In that case the pixel can be brought into the cluster by assigning a high membership function of 0.75 . When $S \Rightarrow \bar{X} Y Z$, it shows that the left end pixel in the neighbourhood window is quite different from the remaining pixels, indicating that it may be an edge pixel. The membership function is assigned $50 \%$ (0.5) as it forms the edge between two clusters. With the assigned membership values the spatial function is calculated using Eq. (25). The new stability-based membership function $u_{i j}^{\mathrm{s}}$, by incorporating the spatial function and control parameters that control the importance of old membership function and spatial function, is (as proposed in [17]) defined as follows:

$$
u_{i j}^{\mathrm{s}}=\frac{u_{i j}^{p} h_{i j}^{q}}{\sum_{k=1}^{c} u_{i j}^{p} h_{i j}^{q}} .
$$

The control of conventional FCM and spatial FCM is done through the parameters $p$ and $q$. The algorithm follows conventional FCM when $p=1, q=0$. The proposed algorithm stability-based Fuzzy C Means (SBFCM) is experimented by giving equal priority $(p=1, q=1)$ to both the membership functions.

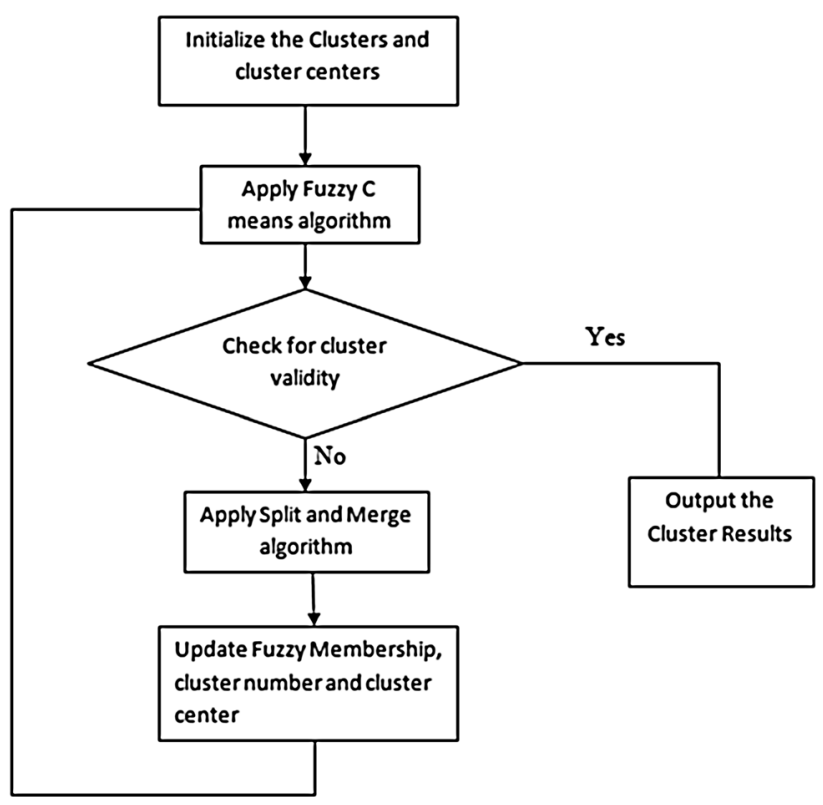

Figure 1. Flowchart of the proposed work. 


\subsection{Stability-based splitting and merging of clusters}

The cluster to be split or merged is identified from the score function $S(i)$ defined by Sun et al [11], which shows that small $S(i)$ indicates that the cluster $i$ tends to contain a large number of pixels with low membership values. The lower the membership value, the farther the object from its cluster centre. Large $S(i)$ indicates strong association of pixels with the cluster. The score function was defined as

$$
S(i)=\frac{\sum_{j=1}^{n} u_{i j}^{\mathrm{s}}}{\text { number of data vectors in cluster } i} .
$$

4.4a Split algorithm Form the ROC around the cluster centre of the chosen cluster $C\{\min (S(i))\}$. The ROA is formed and checked for the stability conditions. The regions of cluster that have ROA similar to ROC indicate that they belong to the same cluster. The ROA that is unstable ( $S \Rightarrow \bar{X} \bar{Y} \bar{Z}$ is satisfied) is chosen as the new ROC $\left(\mathrm{ROC}_{1}\right)$. The centre pixel of the $\mathrm{ROC}_{1}$ forms the new cluster centre for the new cluster. The new clusters arrived (ROC- $C_{1}, \mathrm{ROC}_{1}-C_{2}$ ) are split and cluster number $C$ is updated as $C=C+1$ if and only if $0.9 \geq \frac{\left|C_{1}\right|}{\left|C_{2}\right|} \geq 1.1$.

\section{Merge algorithm}

The ROCs are formed around the cluster centres of the chosen clusters $C\{\max (S(i))\}$. The ROA is formed around both the clusters and the centre pixel of the ROA that has the minimum distance from the two ROCs is chosen as the new cluster centre for the merged clusters. The new cluster number arrived by merging is updated as $C=C-1$ if and only if $0.9 \leq \frac{\left|C_{1}\right|}{\left|C_{2}\right|} \leq 1.1$.

Proposed split and merge algorithm

\section{Results and discussion}

The FCM algorithm integrated with stability to include spatial information and to automatically detect the number of clusters in an image is tested on two synthetic images and two $T_{1}$-weighted brain MRI images. Segmentation is done on original synthetic images and synthetic images corrupted by $9 \%$ and $12 \%$ salt and pepper noise. The algorithm is tested on original MRI images and images corrupted by $5 \%$ and $10 \%$ Gaussian noise. The experiments are done using MATLAB with weighting factor $m=2$ and stopping condition $\varepsilon=0.0001$. The experimental results include the number of clusters, segmented images and validity measures to qualify the segmentation. The validity indexes PC, PE, XB, FS, PA, PD and AIC are calculated to validate the number of clusters found by the algorithm. The segmentation result is validated using the comparison score.

\subsection{Dataset 1 (synthetic image 1)}

A synthetic image with three clusters including the background was taken for experimentation. To test the effect of the proposed algorithm on noisy images, the images corrupted by $9 \%$ and $12 \%$ salt and pepper noise are considered. The measured validation index shows the correct number of clusters as three except in PC, and PA. PC and PA show the number of clusters to be 4 . Thus the optimal number of clusters is correctly classified using the algorithm. The validity measures are tabulated in table 1 and the segmentation results are shown in figure 2. For the image with $6 \%$ noise, except $\mathrm{XB}$ and $\mathrm{PD}$ other validity indexes show correct number of clusters. XB shows 5

(1) Choose $C=2^{n}$ triangular clusters, where $n=1,2,3, \ldots$. Choose the value of $n$ depending on the image under consideration. For heterogeneous images like brain images, high $n$ values are assumed.

(2) Initialize the $C$ cluster centre by finding the centroid of each triangle cluster. Centroid is the point of intersection of the medians of the triangle. The formulas to find the length(s) of the median(s) of a triangle are derived from the law of cosines. $M_{a}=\frac{1}{2} \sqrt{2 c^{2}+2 b^{2}-a^{2}} ; M_{b}=\frac{1}{2} \sqrt{2 c^{2}+2 a^{2}-b^{2}} ; M_{c}=\frac{1}{2} \sqrt{2 a^{2}+2 b^{2}-c^{2}}$. (29)

Update the membership matrix $(U)$ and cluster centres $(V)$ by applying the SBFCM.

3.1. Test for convergence. If convergence is reached then go to step 7, else go to step 4 Compute the score function $S(i)$ :

$$
S(i)=\frac{\sum_{j=1}^{n} u_{i j}^{\mathrm{s}}}{\text { number of data vectors in cluster } i} .
$$

Apply the split and merge algorithm for the clusters with minimum and maximum $S(i)$, respectively.

5.1. Find the cluster $C\{\min (S(i))\}$. Apply the split algorithm if $0.9 \geq \frac{\left|C_{1}\right|}{\left|C_{2}\right|} \geq 1.1$ and update $C=C+1$, else $C$ is unchanged.

5.2. Find the cluster $C\{\max (S(i))\}$. Apply the merge algorithm to merge the cluster with near largest $S(i)$ cluster if $0.9 \leq \frac{\left|C_{1}\right|}{\left|C_{2}\right|} \leq 1.1$ and update $C=C-1$, else $C$ is unchanged.

For the updated number of clusters, perform step 3.

(7)

The new cluster centres and membership functions are given as output.

The flowchart of the proposed algorithm is shown in figure 1. clusters and PA shows 4 clusters. Further, when the algorithm is applied on the image with $12 \%$ noise, PA, FS and 

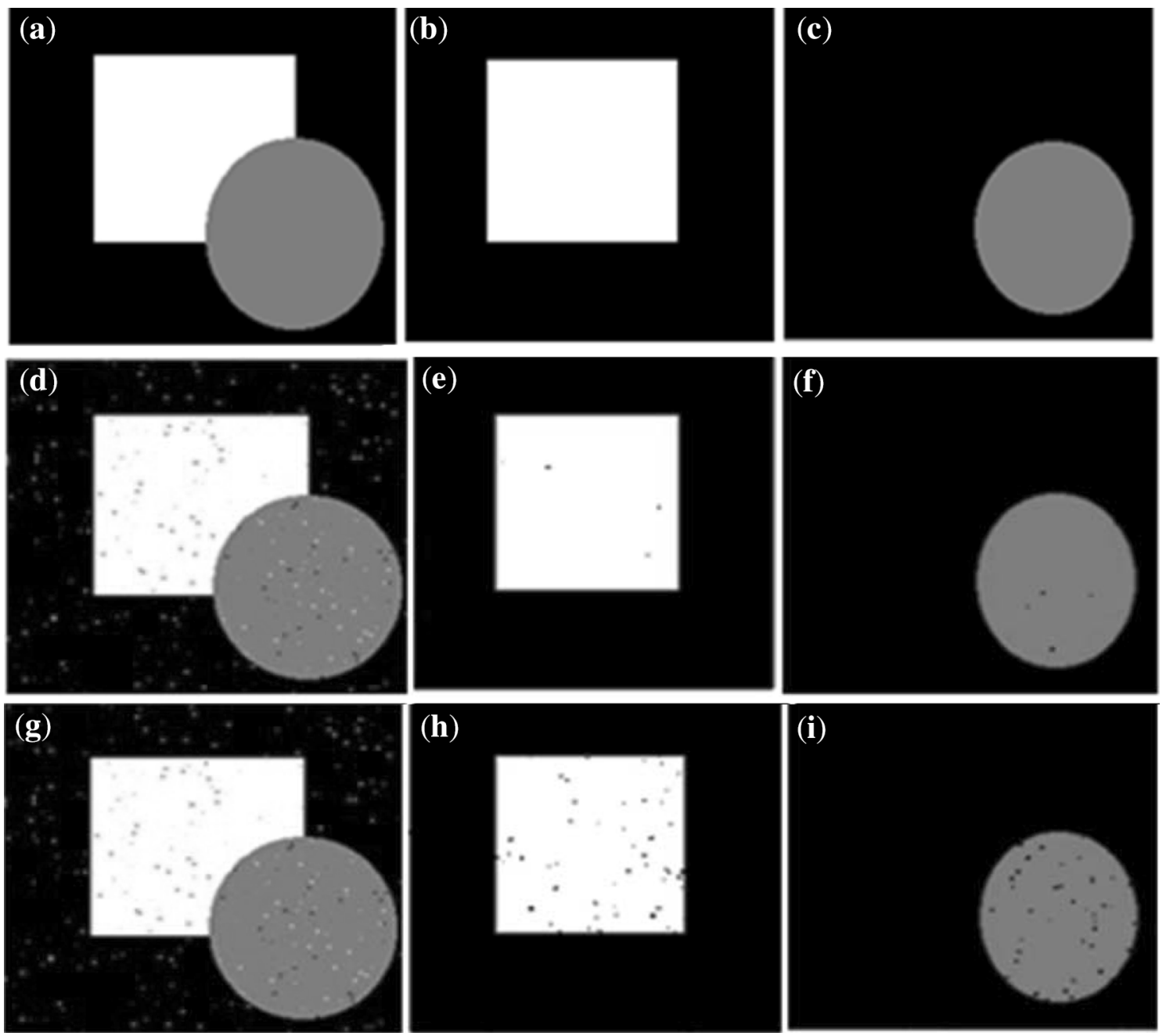

Figure 2. Segmentation of synthetic data 1: (a) $0 \%$ noise level image (i) original, (b) $0 \%$ noise level white region, (c) $0 \%$ noise level grey region, (d) $6 \%$ noise level original image, (e) $6 \%$ noise level white region, (f) $6 \%$ noise level grey region, (g) $12 \%$ noise level original image, (h) $12 \%$ noise level white region and (i) $12 \%$ noise level grey level.

AIC show 3 clusters, whereas PE and XB show 6 clusters. $\mathrm{PD}$ and PC show 5 clusters.

\subsection{Dataset 2 (synthetic image 2)}

The proposed algorithm is tested on a synthetic image with four clusters. The synthetic image with black background, white square, dark grey circle and a light grey circle is considered. When the image is uncorrupted, all but FS and PD show 4 clusters. FS and PD show 5 clusters. For a noise level of $6 \%, \mathrm{PC}, \mathrm{FS}, \mathrm{PD}$ and $\mathrm{AIC}$ give the cluster estimate as 4, whereas $\mathrm{PE}$ and $\mathrm{XB}$ show 5 and PA gives 6. Furthermore, for this dataset with $12 \%$ noise, four indexes show 4 clusters. XB, FS, PD and AIC indicate 4 clusters, PA gives 6 clusters and the remaining two show 5 clusters. Figure 3 shows the clustering of synthetic image 2 for the three different noise levels. The validity indexes are consolidated in table 1 .

\subsection{Dataset 3 (brain data 1)}

The segmentation of synthetic image has shown results in the literature. But for real-time images like brain MRI the number of clusters is unknown initially. Normally, brain images are segmented with respect to three main regions GM, WM and CSF. In these cases, the number of clusters can be easily predetermined as 6 clusters that include WM, GM, CSF, fat, bone and air. However, in case of tumour or lesions the number of clusters cannot be predetermined. In diseases like Focal Cortical Dysplasia (FCD), a common malformation of cortical development, the cortical lesions 

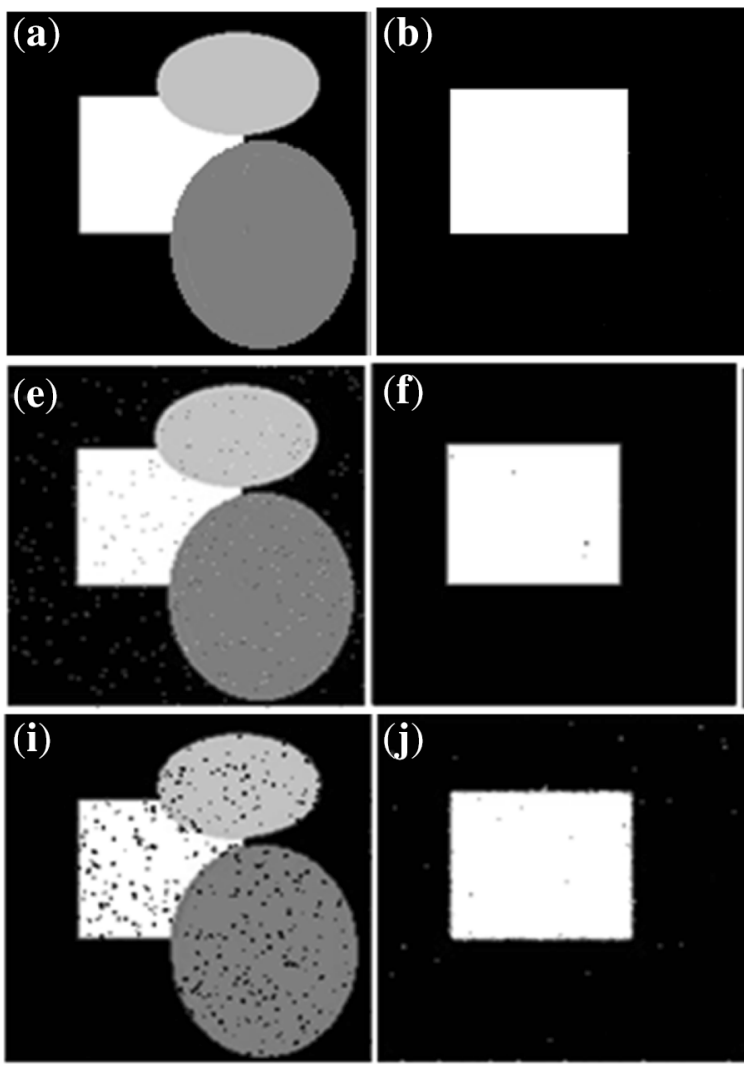
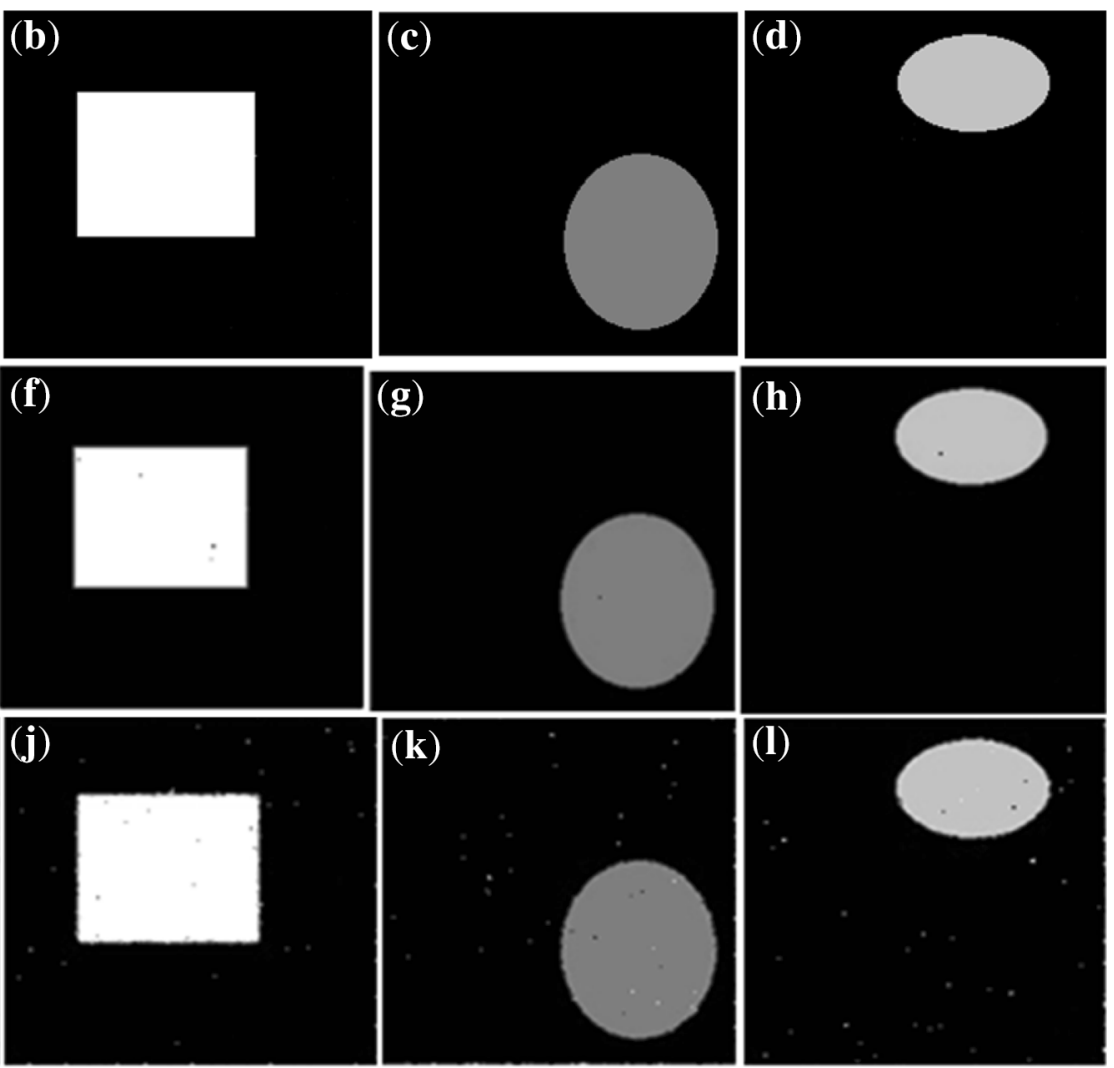

Figure 3. Segmentation of synthetic data 2: (a) $0 \%$ noise level original, (b) $0 \%$ noise level white region, (c) $0 \%$ noise level grey region 1, (d) $0 \%$ noise level grey region 2, (e) 5\% noise level original image, (f) 5\% noise level white region, (g) 5\% noise level grey region 1, (h) 5\% noise level grey region 2, (i) 12\% noise level original image, (j) 12\% noise level white region, (k) $12 \%$ noise level grey region 1 and (l) $12 \%$ noise level grey region 2 .

are not easily identified. Especially in early stages, the diagnosis of FCD is difficult. Brain data 1 is a $T_{1}$-weighted image, which consists of lesion indicated by the arrow. The FCD lesion is too subtle to be visualized and cannot be segmented by usual means.

Experts of relevant field are required to identify the lesions in both pre- and post-surgical cases. Hence, in such cases, the number of clusters cannot be predetermined for segmentation using FCM. Hence, an automation of FCM to determine the number of clusters is necessary. This algorithm is proposed to detect the number of clusters automatically. The number of clusters in brain MRI data is 12 , including the fat, bone and air. Figure 4 shows the segmented results of brain data with $0 \%, 5 \%$ and $10 \%$ Gaussian noise. The GM, WM and lesions are shown separately.

The validity indexes indicate the correct number of clusters in 5 cases except FS and PD. In this case, FS shows 13 clusters and PD indicates 11 clusters. The remaining indexes show 5 clusters. When the image is subjected to $5 \%$
Gaussian noise, though the indexes PC and PA indicate 12 clusters, the indexes PE, XB, FS, PD and AIC show the exact cluster number 10. Further, when the algorithm is tested for image with $10 \%$ Gaussian noise, segmentation is seen to be good. FS, PA and AIC indexes show the number of clusters to be 10. The number of indexes as per PC and $\mathrm{XB}$ is $14, \mathrm{PE}$ is 15 and $\mathrm{PD}$ is 11 . The segmentation results are good even in the presence of noisy pixels.

\subsection{Dataset 4 (brain data 2)}

Brain data 2 consists of two lesions, one each in posterior ends. The segmentation of WM, GM and the lesion are shown in figure 5 . As shown in table 1 , most indexes gave optimal cluster number 12 except $\mathrm{PD}, \mathrm{PC}$ and $\mathrm{XB}$ for the original image. The FS, PE, PC and AIC indicate that 10 is the optimal cluster number for $5 \%$ noise dataset, which matches the structure of the image. The PA and XB indexes 

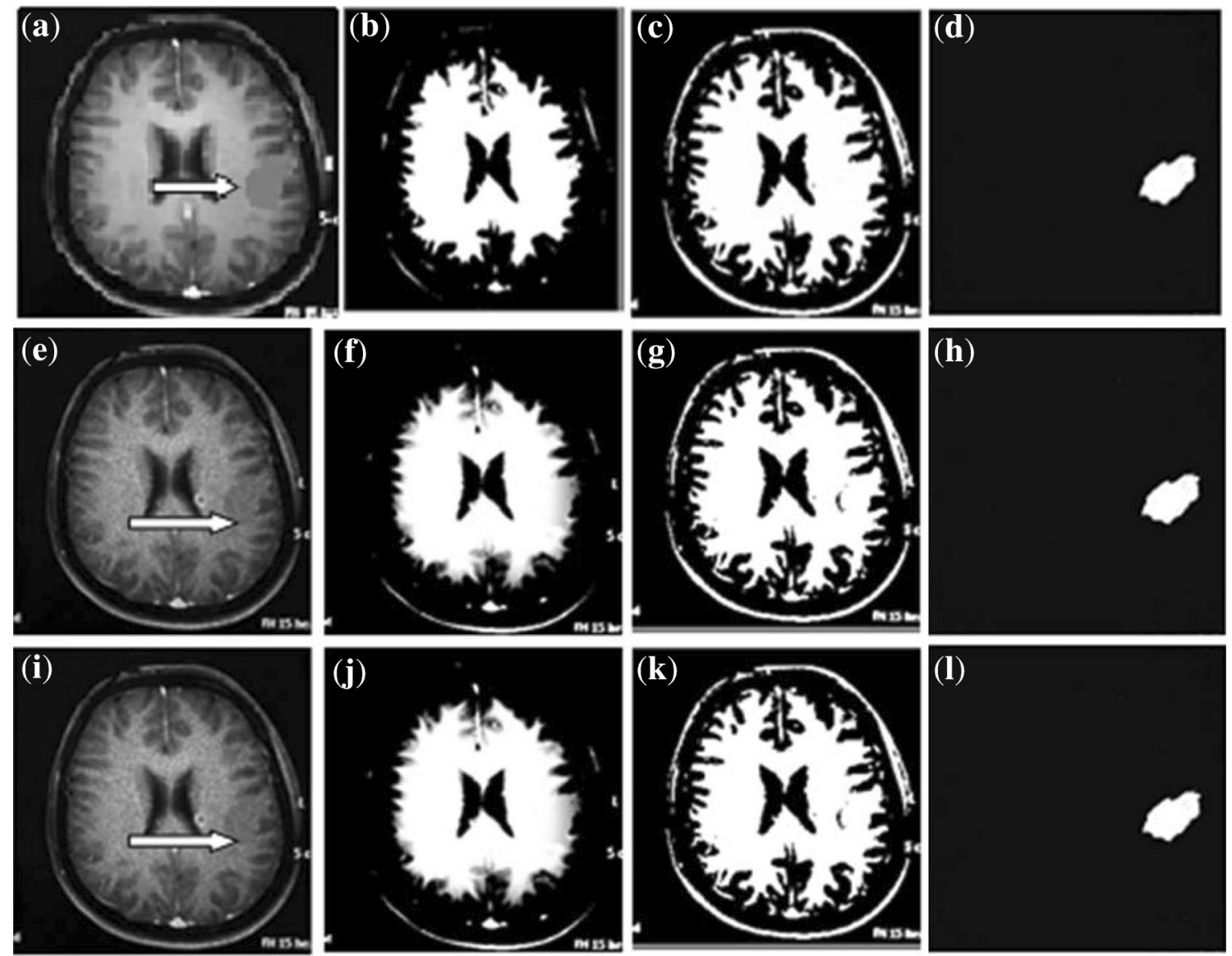

Figure 4. Segmentation of brain MRI data 1: (a) 0\% noise level original, (b) 0\% noise level white matter, (c) $0 \%$ noise grey matter, (d) $0 \%$ noise level lesion, (e) 5\% noise level original image, (f) 5\% noise level white matter, (g) 5\% noise level grey matter, (h) 5\% noise level lesion, (i) 10\% noise level original image, (j) 10\% noise level white matter, (k) 10\% noise level grey matter and (l) $10 \%$ noise level lesion.

considered that 16 is the optimal cluster number. However, $\mathrm{PD}$ index considered 14 to be the best cluster number. For $10 \%$ noise of dataset, only PD, XB and AIC indexes gave the actual cluster number. PC and FS considered 16 clusters, PE shows 14 and PA shows 15.

\subsection{Comparison of validity indexes vs number of clusters}

The values of the afore-discussed validity indexes are shown in table 2. The validity indexes of the four different datasets are obtained for different noise levels.

The table shows that if the correct number of clusters is identified, the value of PC nears unity or scatters near 0 for misclassified clusters. In case of PE, the index value is near
0 for correct clusters and near 1 for wrong cluster identification. From the table it is seen that XB, FS and AIC values are low for compact clusters and high for PA and PD. The variations of validity index and the number of clusters are shown in figure 6.

From figure 6 and tables 1 and 2 it is clear that the validity index AIC determines the correct number of clusters in all datasets under the conditions of different noise levels. Other validity indices show correct cluster number in some cases and wrong number of clusters in some cases. The segmentation accuracy is calculated using (17) and the comparison score for FCM, KFCM [7], FBSA [11] and the proposed method is tabulated in table 3 .

The table shows that the proposed method improves the segmentation accuracy when compared with the other methods in the presence of noise and outliers. The results have been shown for various noise levels, and it is seen that 

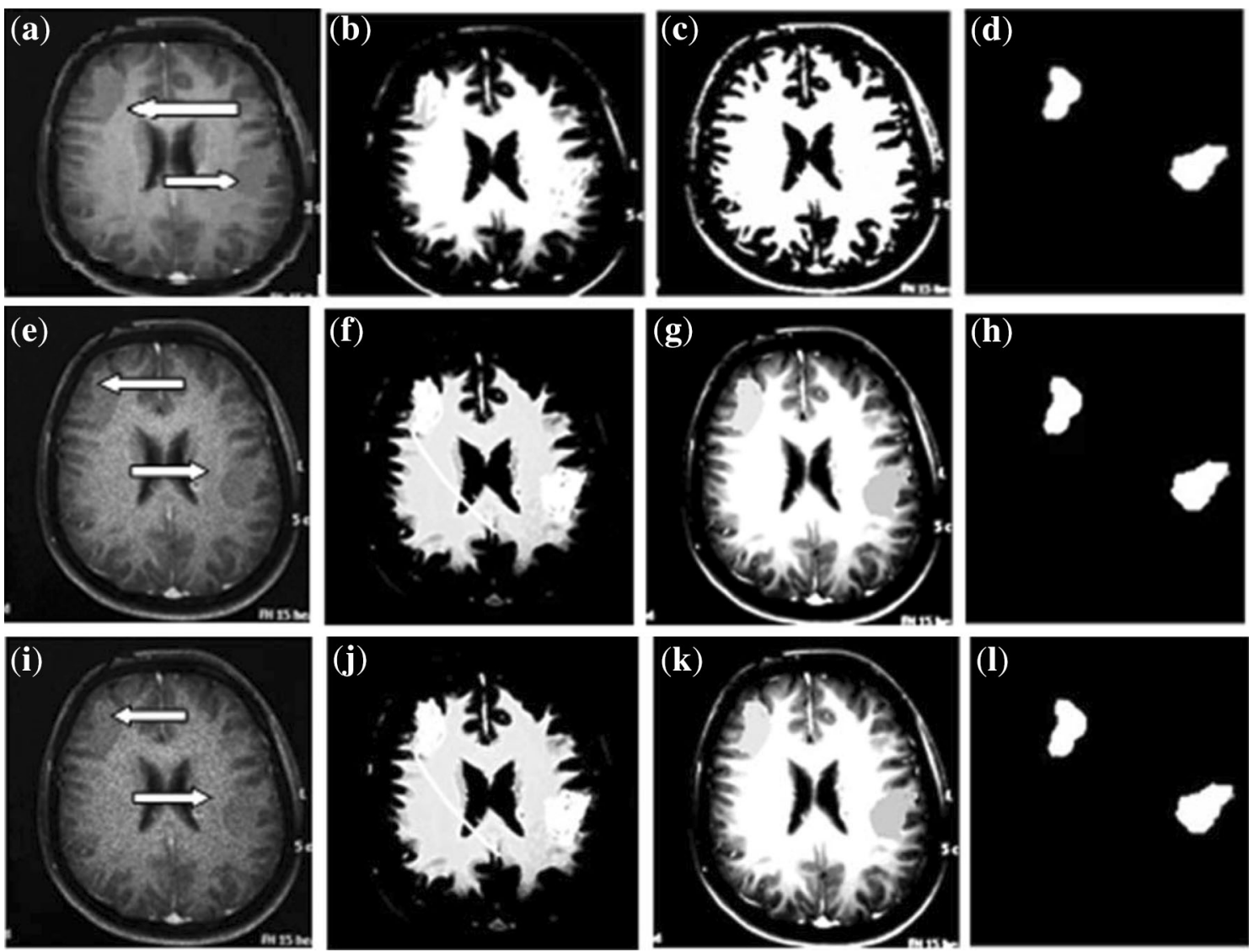

Figure 5. Segmentation of brain MRI data 2: (a) 0\% noise level original, (b) 0\% noise level white matter, (c) $0 \%$ noise grey matter, (d) $0 \%$ noise level lesion, (e) $6 \%$ noise level original image, (f) 5\% noise level white matter, (g) 5\% noise level grey matter, (h) 5\% noise level lesion, (i) 10\% noise level original image, (j) $10 \%$ noise level white matter, (k) $10 \%$ noise level grey matter and (l) $10 \%$ noise level lesion.

for higher noise levels the segmentation is better than in standard FCM and other methods, but at $0 \%$ noise level, the comparison scores of the proposed method is less than those of the original FCM. This is due to the effect of the spatial membership function proposed in Eq. (27), involving the modified Sylvester's theorem. A portion of pixel array of an image is shown in figure 7 .

The region shown in the green box represents the centre pixel to be an edge signal. When we apply the stability criteria as in section 4.2 , the centre pixel 252 will be considered as an edge pixel. When the next region (region above the green box) is considered, pixel 255 is considered as the edge pixel and when the region adjacent to the green box is considered, pixel 249 is considered as the edge signal. However, when the clusters are formed, pixel 252 will be taken as the edge and two pixels 255 and 249 will be left out of the clusters. Hence there will be a pixel deviation when compared with the actual result of FCM. This results in lesser segmentation accuracy of the proposed method when compared with FCM. A portion of a segmented image by FCM and proposed method is shown in figure 8.

From figure 8 it is seen that the proposed method shows a pixel difference at the edges on comparing the segmentation results of the FCM and proposed method. However, this does not have much impact as there is only a pixel difference along the edges. Hence the reduction in segmentation accuracy when compared with FCM may be disregarded. However, at high noise levels, the proposed method increases the spatial function, thereby improving the clusters that result in higher comparison scores. The noisy pixels in a region would have been grouped under a different cluster in the case of FCM. However, the proposed method includes it in the appropriate cluster based on the modified spatial function and membership function, thereby increasing the comparison score. Moreover, the clustering is done automatically, which is an added advantage. 
(a)

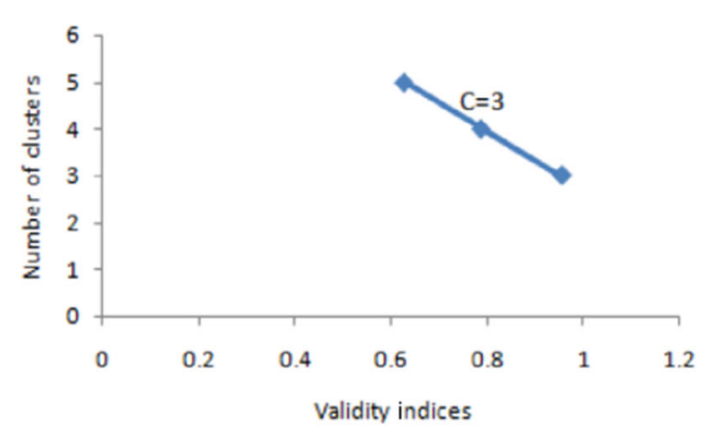

PC

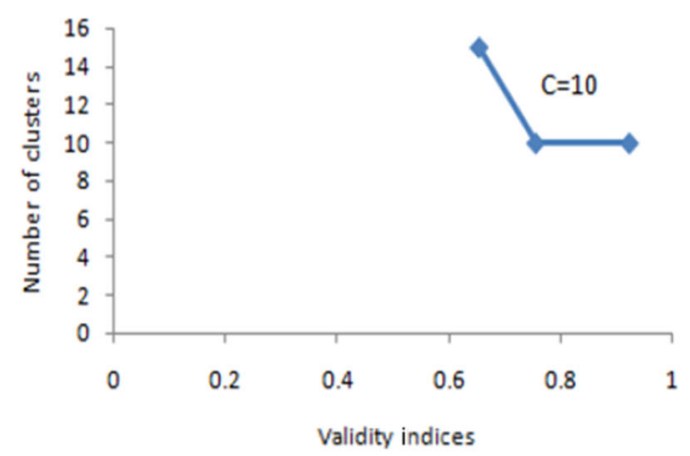

(b)

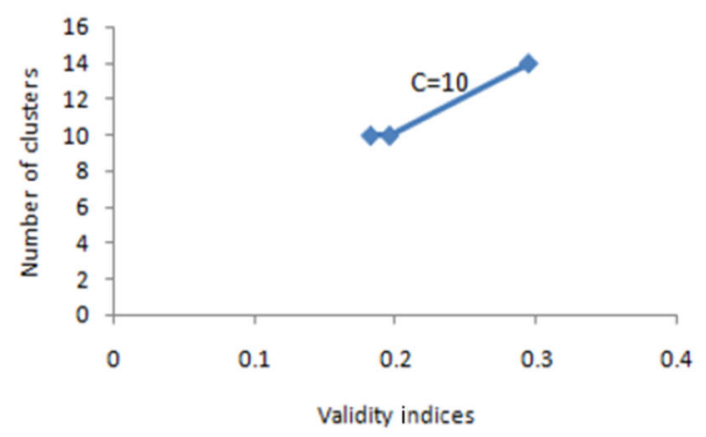

PE

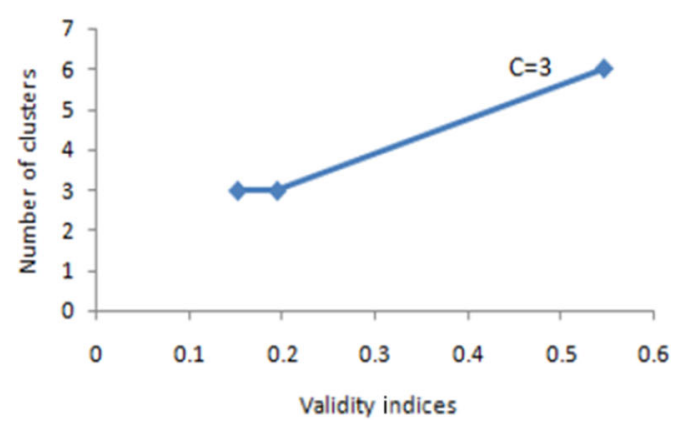

PC

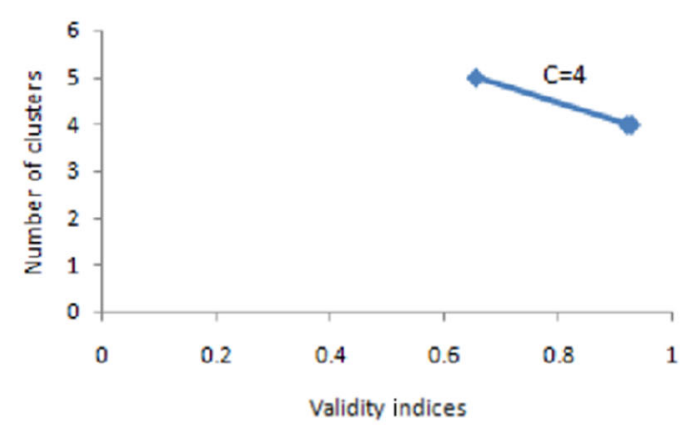

PC

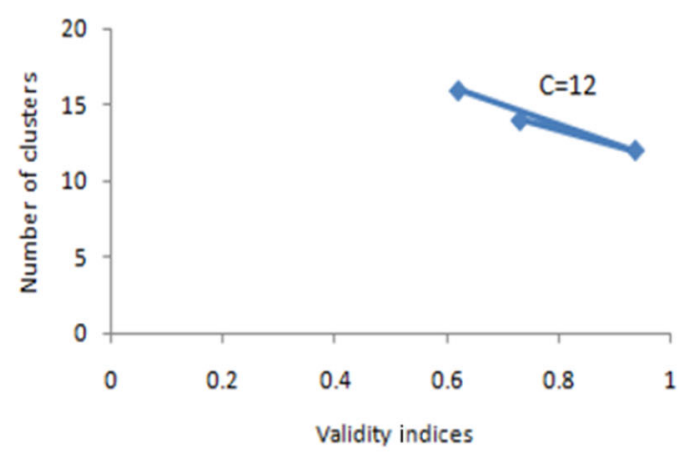

PE

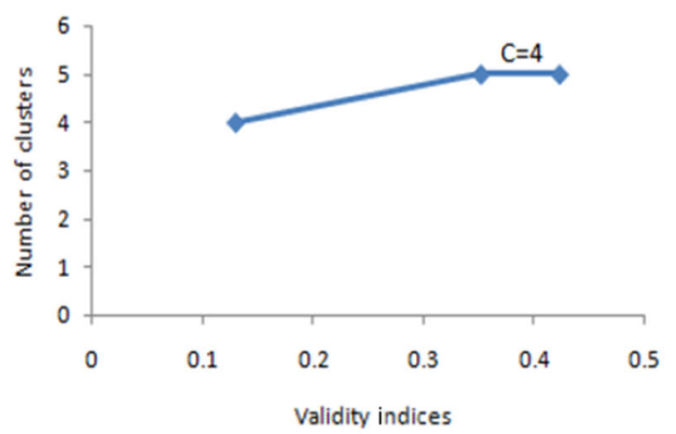

PE

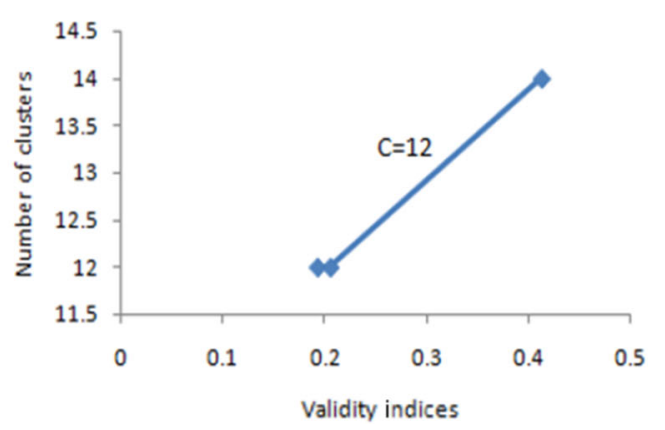

Figure 6. (a) Validity index vs cluster number for PC. (b) Validity index vs cluster number for PE. (c) Validity index vs cluster number for XB. (d) Validity index vs cluster number for FS. (e) Validity index vs cluster number for PA. (f) Validity index vs cluster number for PD. (g) Validity index vs cluster number for AIC. 
(c)

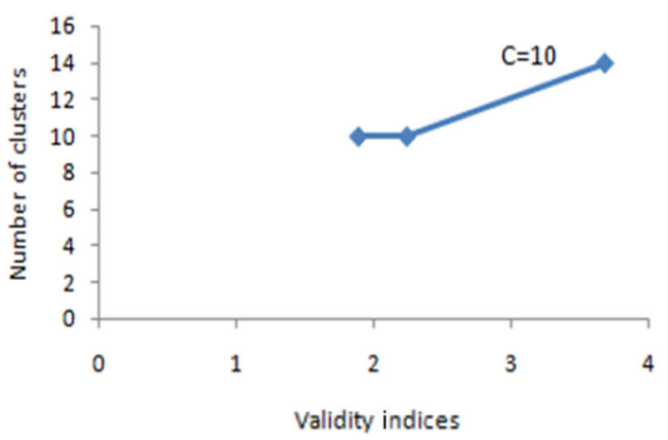

XB

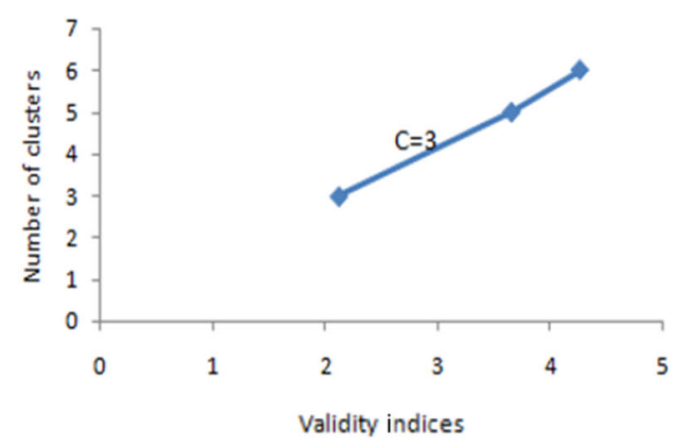

(d)

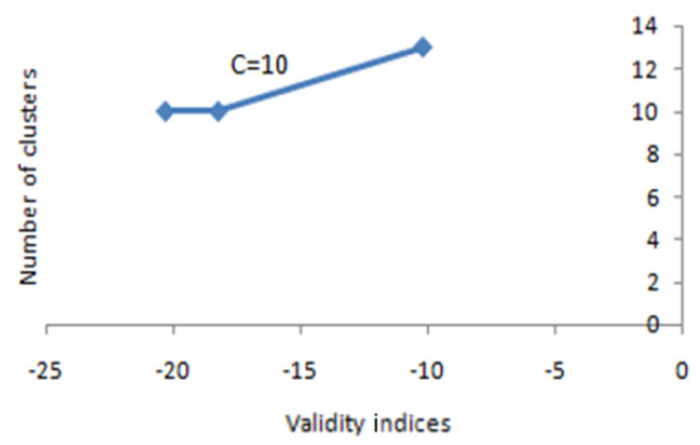

FS

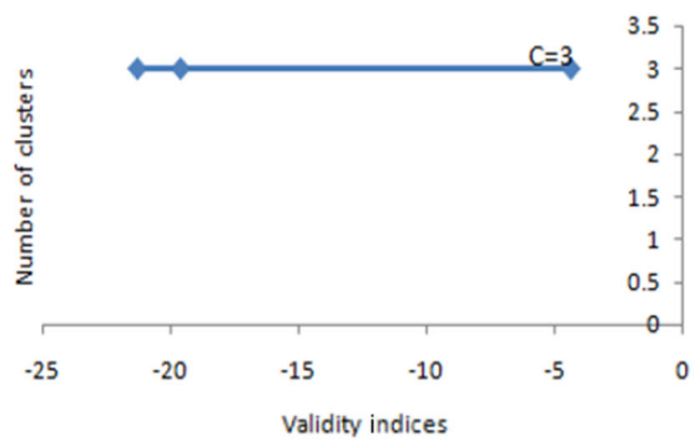

XB

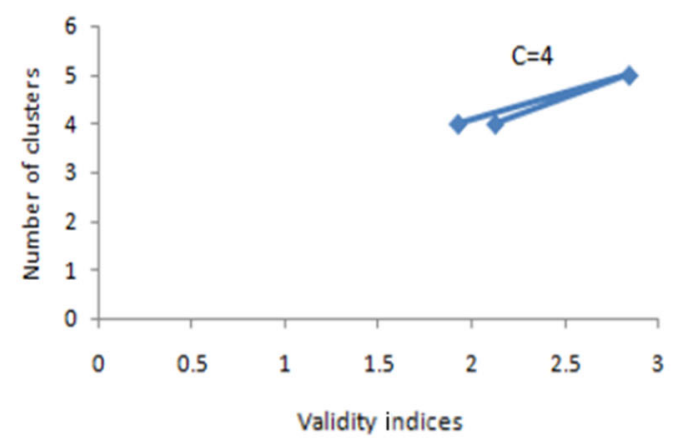

XB

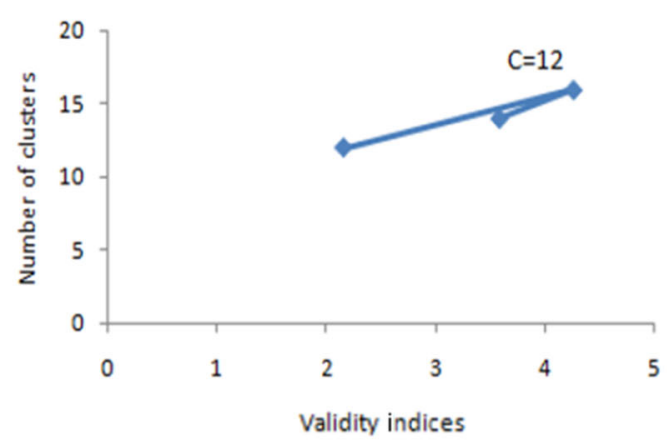

FS

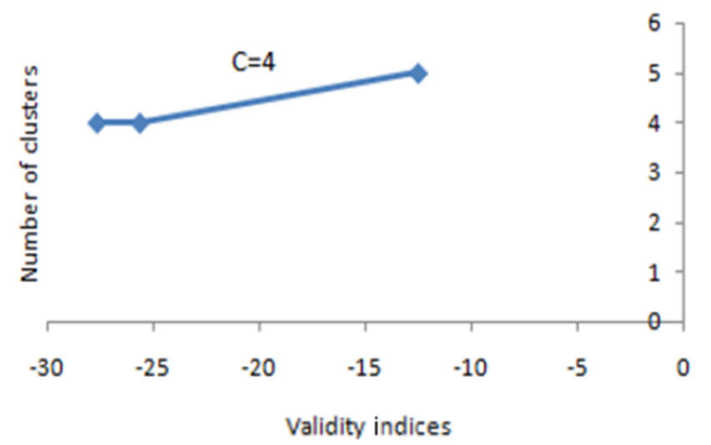

FS

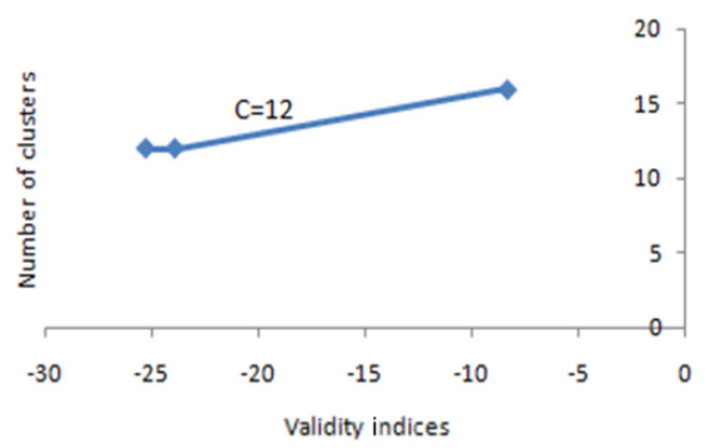

Figure 6. continued 
(e)

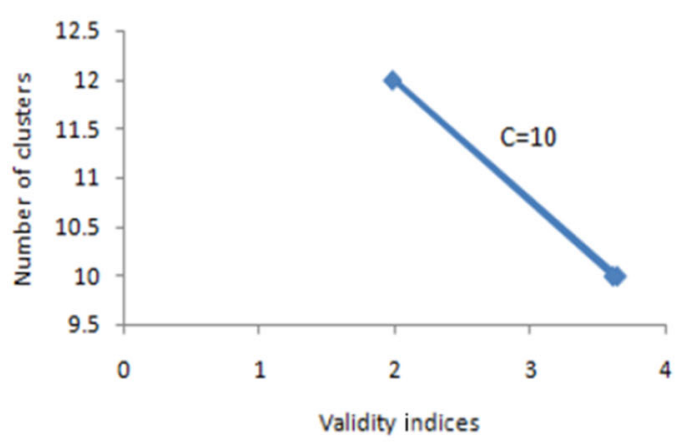

PA

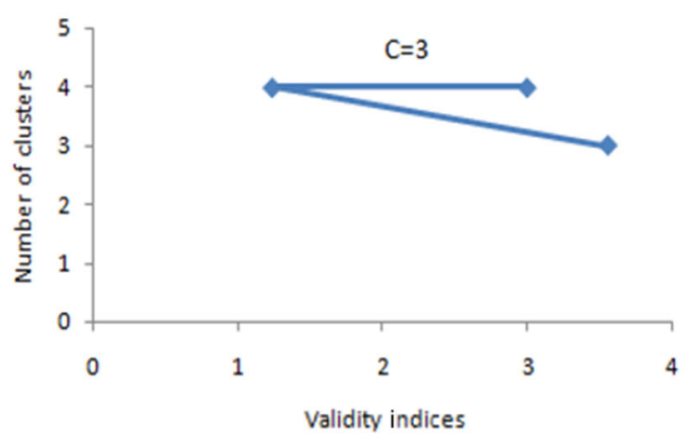

(f)

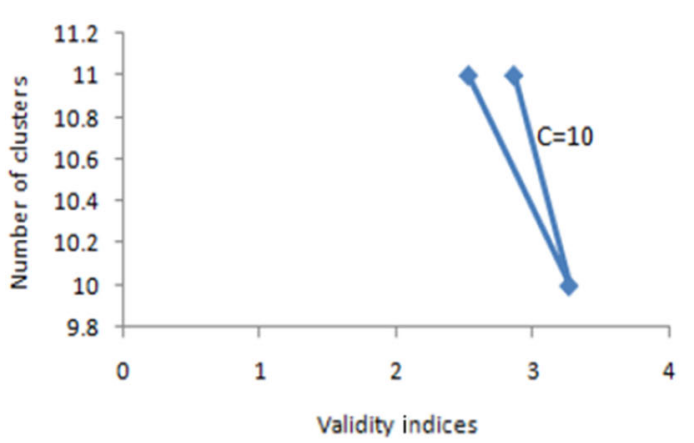

PD

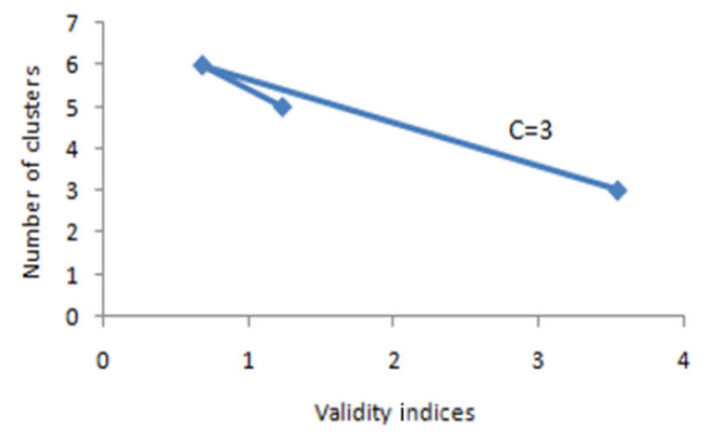

PA

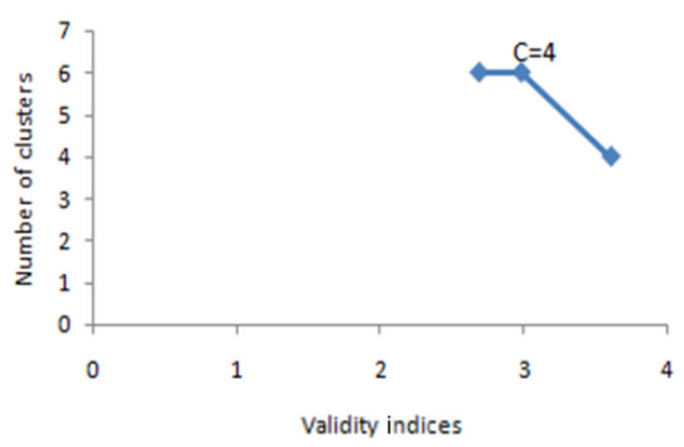

PA

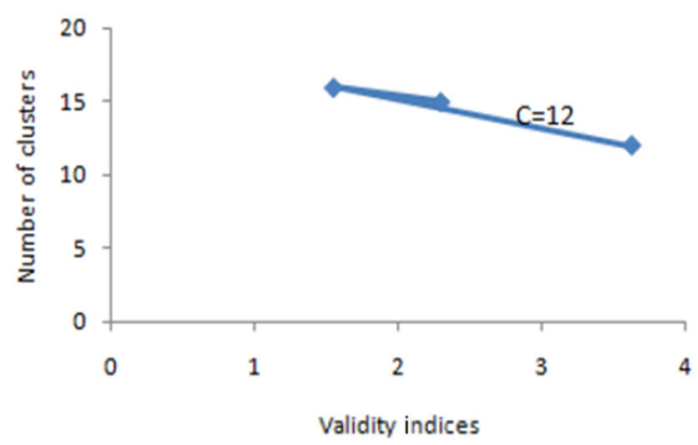

PD

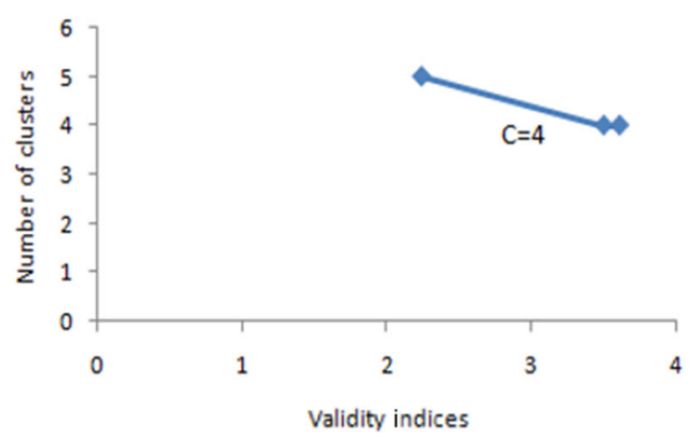

PD

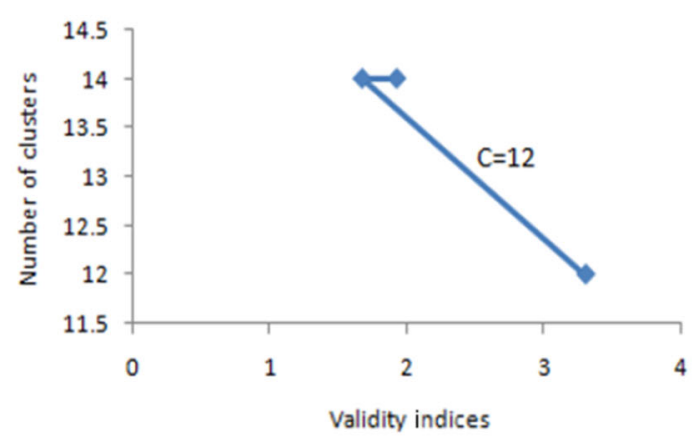

Figure 6. continued 

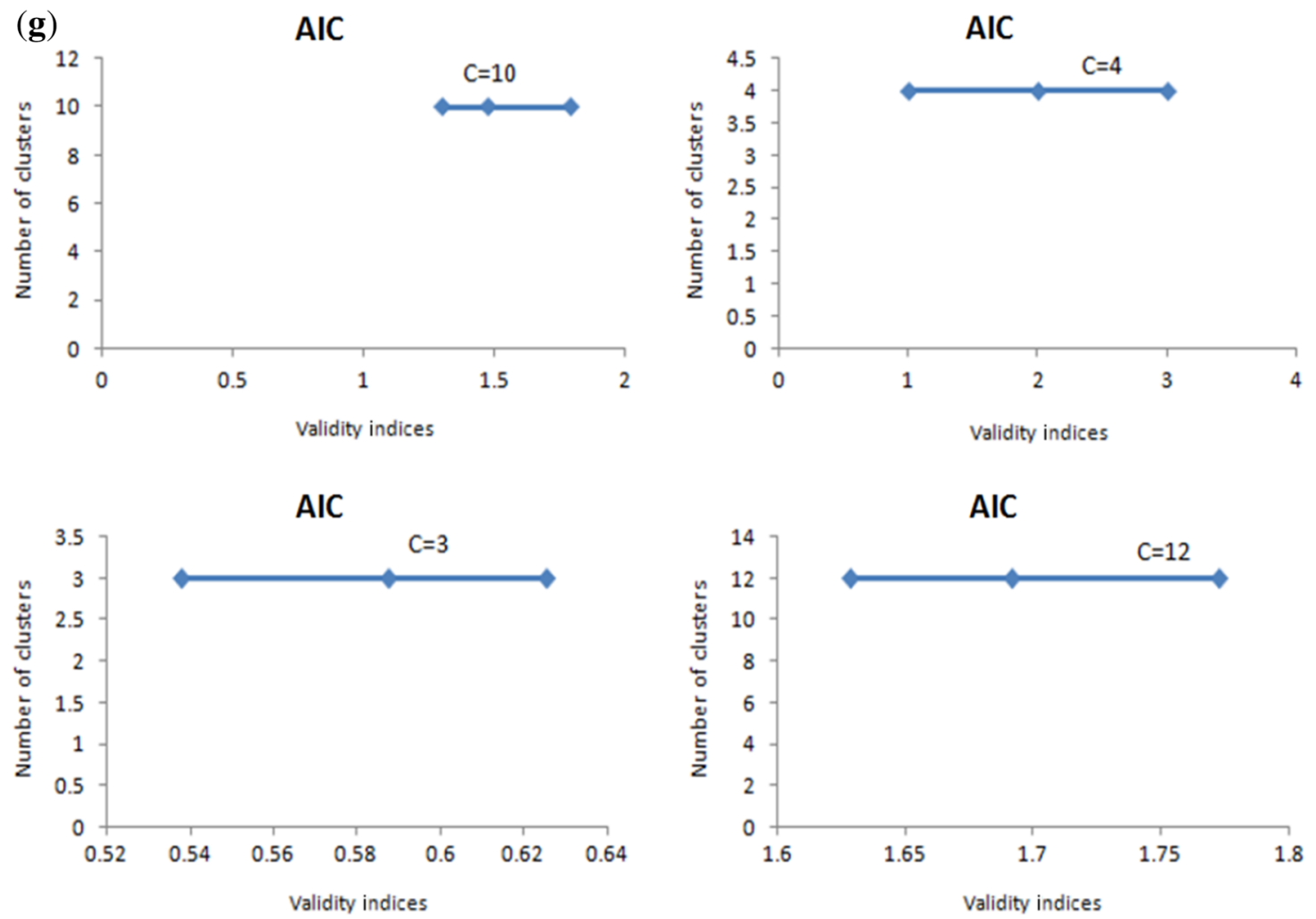

Figure 6. continued

$$
\left[\begin{array}{cccccccccc}
11 & 2 & 23 & 14 & 15 & 21 & 15 & 15 & 13 & 13 \\
14 & 11 & 13 & 16 & 13 & 16 & 22 & 16 & 12 & 12 \\
13 & 12 & 10 & 12 & 248 & 251 & 253 & 13 & 15 & 14 \\
12 & 18 & 255 & 252 & 255 & 253 & 252 & 248 & 14 & 15 \\
12 & 249 & 252 & 254 & 22 & 251 & 255 & 246 & 13 & 10 \\
238 & 254 & 253 & 251 & 250 & 252 & 254 & 251 & 253 & 249 \\
245 & 248 & 252 & 249 & 255 & 255 & 256 & 253 & 252 & 253 \\
248 & 245 & 251 & 248 & 254 & 256 & 255 & 250 & 16 & 251 \\
252 & 252 & 249 & 247 & 255 & 255 & 253 & 249 & 254 & 250 \\
254 & 254 & 247 & 250 & 253 & 251 & 252 & 247 & 252 & 251
\end{array}\right]
$$

Figure 7. Pixel array for a certain portion of the image.
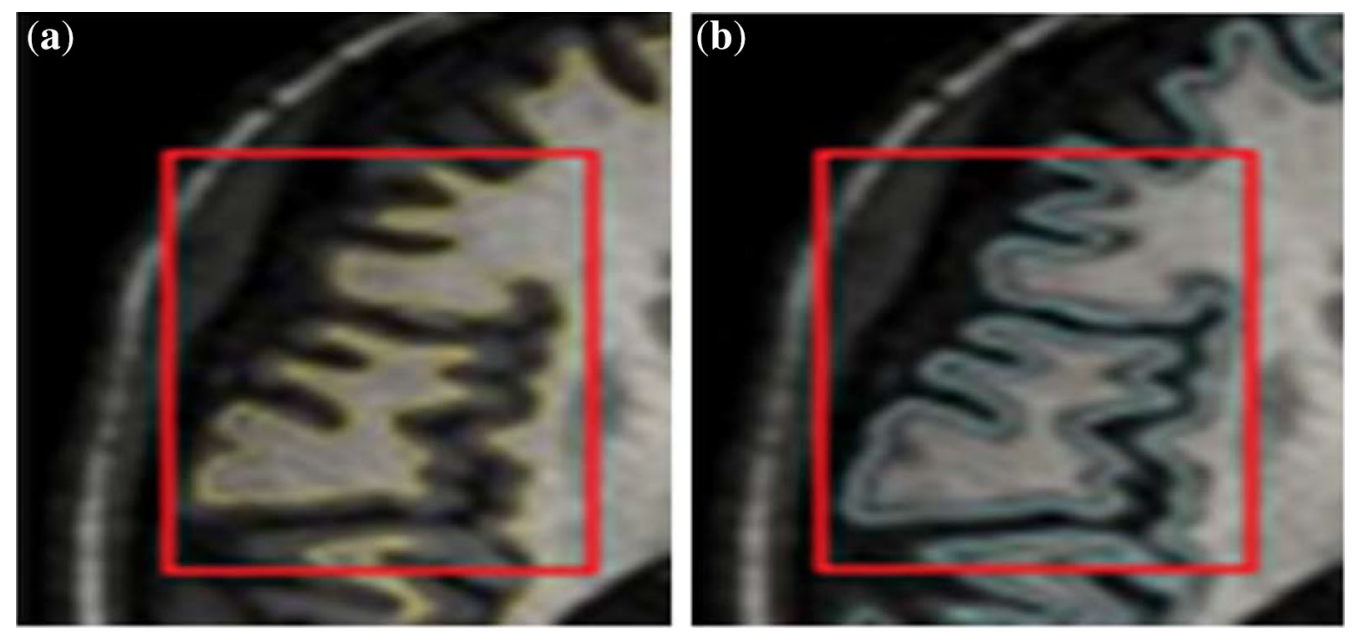

Figure 8. Edge segmentation (zoomed image) by (a) FCM and (b) proposed method. 


\section{Conclusion}

In the proposed work, the basic FCM is integrated with stability concept to determine the number of clusters, making it insensitive to noise. The split and merge algorithm is incorporated in the FCM algorithm, to determine the number of clusters automatically. The modified Sylvester's theorem is applied in the split and merge algorithm to make corrections for the noisy pixels, thus making it robust to noise automatically. The proposed work is tested on synthetic and brain MRI images and qualitative and quantitative results show good segmentation without manual intervention. This encourages fully automatic FCM segmentation of images even in noisy images. The split and merge algorithm does not involve distance calculation at each iteration, but the application of modified Sylvester's theorem at each iteration increases the time consumption. Still improvements made with respect to automation and noise sensitivity together increases the overall computational speed.

\section{References}

[1] Pappas T N 1992 An adaptive clustering algorithm for image segmentation. IEEE Trans. Signal Process. 40: 901-914

[2] Liew A W C, Leung S H and Lau W H 2000 Fuzzy image clustering incorporating spatial continuity. IEEE Proc. Vision Image Signal Process. 147: 185-192

[3] Szeto L K, Liew A W C, Yan H and Tang S S 2003 Gene expression data clustering and visualization based on a binary hierarchical clustering framework. J. Visual Lang. Comput. 14: 341-362 (special issue on "Biomedical Visualization for Bioinformatics")

[4] Wu S H, Liew A W C, Yan H and Yang M S 2004 Cluster analysis of gene expression data based on self-splitting and merging competitive learning. IEEE Trans. Inf. Technol. Biomed. 8: 5-15

[5] Bezdek J C 1998 Pattern recognition in handbook of fuzzy computation. IOP Publishing Ltd., Bristol, UK, Chapter F6

[6] Pham D L and Prince J L 1999 Adaptive fuzzy segmentation of magnetic resonance images. IEEE Trans. Med. Imaging 18: 737-751

[7] Zanaty E A, Aljahdali S and Debnath N 2009 A kernelized fuzzy c-means algorithm for automatic magnetic resonance image segmentation. J. Comput. Methods Sci. Eng. 1: 123-136

[8] Yu Z, Au O C, Zou R, Yu W and Tian J 2010 An adaptive unsupervised approach toward pixel clustering and color image segmentation. Pattern Recogn. 43: 1889-1906

[9] Gong M, Liang Y, Shi J, Ma W and Ma J 2013 Fuzzy $\mathrm{C}$-means clustering with local information and kernel metric for image segmentation. IEEE Trans. Image Process. 22: 573-584

[10] Zhang Y J and Liu Z Q 2002 Self-splitting competitive learning: a new on-line clustering paradigm. IEEE Trans. Neural Netw. 13: 369-380

[11] Sun H, Wang S and Jiang Q 2004 FCM-based model selection algorithms for determining the number of clusters. Pattern Recogn. 37: 2027-2037
[12] Lin C R and Chen M S 2005 Combining partitional and hierarchical algorithms for robust and efficient data clustering with cohesion self-merging. IEEE Trans. Knowl. Data Eng. 17: 145-159

[13] Liu J and Ramamohanarao K 2007 Multiple self-splitting and merging competitive learning algorithm. Advances in Knowledge Discovery and Data Mining, Lecture Notes in Computer Science, vol. 4426, pp. 704-711

[14] Yang M S and Lai C Y 2011 A robust automatic merging possibilistic clustering method. IEEE Trans. Fuzzy Syst. 19: 26-41

[15] Fa R and Nandi A K 2012 SMART: novel self-splittingmerging clustering algorithm. In: Proceedings of the 20th European Signal Processing Conference (EUSIPCO 2012), Bucharest, Romania, pp. 27-31

[16] Zanaty E A 2012 Determining the number of clusters for kernelized fuzzy C-means algorithms for automatic medical image segmentation. Egyptian Informat. J. 13: 39-58

[17] Chuang K S, Tzeng H L, Chen S, Wu J and Chen T J 2006 Fuzzy c means clustering with spatial information for image segmentation. Comput. Med. Imaging Graph. 30: 9-15

[18] Zhao F, Jiao L and Liu H 2011 Fuzzy c-means clustering with non-local spatial information for noisy image segmentation. Front. Comput. Sci. China 5: 45-56

[19] Zaixin Z, Lizhi C and Quan C G 2014 Neighbourhood weighted fuzzy c-means clustering algorithm for image segmentation. IET Image Process. 8: 1751-9659

[20] Zhang H, Wu Q M J, Zheng Y, Nguyen T M and Wang D C 2014 Effective fuzzy clustering algorithm with Bayesian modeland mean template for image segmentation. IET Image Process. 8: 571-581

[21] Yanling L and Yi S 2010 Fuzzy c-means clustering based on spatial neighborhood information for image segmentation. $J$. Syst. Eng. Electron. 21: 323-328

[22] Ogata K 2010 Modern control engineering, $5^{\text {th }}$ ed. Prentice Hall, Englewood Cliffs

[23] Gopal M 1993 Modern control system theory. New Age International Publishers, New Delhi

[24] Bezdek J C, Ehrlich R and Full W 1984 FCM: fuzzy c means algorithm. Comput. Geosci. 10: 191-203

[25] Xie X L and Beni G 1991 A validity measure for fuzzy clustering. IEEE Trans. Pattern Mach. Intell. 13: 841-847

[26] Fukuyama Y and Sugeno M 1989 A new method of choosing the number of clusters for the fuzzy c means method. In: Proceedings of the Fifth Fuzzy System Symposium, pp. 247-250

[27] Davies D L and Bouldin D W 1979 A cluster separation measure. IEEE Trans. Pattern Mach. Intell. 1: 224-227

[28] Gath Geva A B 1989 Unsupervised optimal fuzzy clustering. IEEE Trans. Pattern Mach. Intell. 11: 773-881

[29] Andre T, Antonini M, Barlaud M and Gray R M 2007 Entropy-based distortion measure and bit allocation for wavelet image compression. IEEE Trans. Image Process. 16: 3058-3064

[30] Masulli F and Schenone A 1999 A fuzzy clustering based segmentation system as support to diagnosis in medical imaging. Artif. Intell. Med. 16: 29-47

[31] Nirmala S and Royna Daisy V 2013 A novel segmentation approach by the concept of stability analysis. Int. J. Appl. Inf. Syst., ICWAC-2013, pp. 17-22 